\title{
Transparentalités contemporaines
}

\section{Laurence Hérault, Pr anthropologie, Aix-Marseille Université}

Nous sommes désormais familiers du terme homoparentalité qui a débordé largement la sphère des publications en sciences sociales pour s'inviter dans les débats publics, les manifestations dominicales et les conversations ordinaires, mais nous le sommes beaucoup moins du terme transparentalité tout juste émergeant. Il s'applique généralement aux familles où l'un des parents au moins est une personne transgenre. Comme pour homoparentalité, ce terme est efficace pour évoquer, sans circonvolutions inutiles, l'expérience parentale des personnes trans, de même que leur famille. C'est pourquoi il a toutes les raisons de devenir rapidement un terme courant du vocabulaire contemporain. Pourtant avant de l'adopter définitivement, il n'est pas inutile d'en questionner quelques implicites. Tout d'abord sa nouveauté même ne doit pas nous amener à penser qu'il y a là une réalité nécessairement nouvelle. La médiatisation des personnes trans qui a longtemps balancé entre les icônes féminines issues du cabaret et la figure de la prostituée du Bois de Boulogne a non seulement masqué la diversité des parcours des personnes trans mais a surtout invisibilisé leur inscription familiale et parentale : marginalisées voire stigmatisées, il n'était même pas envisageable de penser que ces personnes puissent avoir des enfants et fonder une famille. L'expérience parentale des personnes trans est donc vraisemblablement ancienne même s'il est indéniable qu'elle bénéficie aujourd'hui d'une ouverture inédite (du fait de la banalisation même de la transition) et de possibilités accrues de réalisation via les PMA (insémination avec donneur, autoconservation des gamètes avant opération, etc.). Le terme transparentalité pose également, par ailleurs, une question plus fondamentale. Son usage doit-il amener à penser que la parentalité trans est a priori une forme « autre » de parentalité. Autrement dit, y a-t-il effectivement là une forme familiale particulière, une parentalité voire une parenté spécifique ? Les familles transparentales sont-elles à la fois différentes des autres et semblables entre elles ? Même si les travaux nous manquent encore en la matière, ce serait sans doute une erreur de le penser car ce qui détermine cette dénomination c'est l'expérience de la transition de l'un des parents (ou des deux). Or celle-ci peut intervenir à différents moments d'une histoire familiale (avant, après ou entre la naissance des enfants) et être associée à des formes conjugales variées (homosexuelle ou hétérosexuelle), donc une famille transparentale peut être hétéroparentale, homoparentale, monoparentale ou recomposée. Les configurations familiales trans sont donc non seulement multiples mais aussi loin d'être sans «commune mesure » avec les autres. Ce qui ne veut pas dire non plus que l'expérience familiale des personnes trans ne comporte pas des singularités, que ces parents ne vivent pas des choses que les autres parents ne vivent pas, de même que leurs partenaires ou leurs enfants. En ce sens, il est important de saisir ce qu'une transition fait à une famille et aussi ce qu'une famille fait à une transition dans un contexte social où l'on ne peut que constater que cette association n'est pas envisagée comme allant de soi. Longtemps en effet les spécialistes du transsexualisme ont considéré qu'il y avait une incompatibilité entre le fait d'avoir des enfants et le fait de réaliser une transition. En 1969, R. Green et J. Money suggèrent, par exemple, qu'il est préférable d'éloigner définitivement les jeunes enfants de leur parent trans de façon à ce qu'ils ne soient pas perturbés par leur transition. De même jusqu'au milieu des années 2000, l'une des équipes hospitalières françaises chargées des « troubles de l'identité de genre » (TIG) faisait de la parentalité un critère d'exclusion du protocole (Cordier, Chiland \& Gallarda, 2001). 
Bien que ces avis aient été révisés notamment au regard de l'expérience clinique ${ }^{1}$, il reste que dans nombre de lieux contemporains, médicaux et non médicaux, la parentalité et la transidentité sont vues comme des expériences incompatibles ou difficilement compatibles. Et ce, à tel point qu'on peut se demander s'il ne faudrait tout simplement pas entendre le terme transparentalité autrement. Si l'ajout du préfixe trans peut faire sens, décrivant une réalité spécifique, ne serait-ce pas dans la mesure où la légitimité parentale des personnes trans n'est pas a priori assurée voire même est, ou peut être, contestée ? Quoi qu'il en soit, cette interrogation sur la catégorie même de transparentalité n'est pas anodine parce qu'elle suppose que lorsque nous nous intéressons à l'expérience parentale des personnes trans, il faut non seulement prendre garde à la rendre dans sa diversité même mais aussi à la replacer dans son contexte : on ne peut appréhender leur expérience sans la rapporter à la manière dont ces familles sont socialement pensées et admises. C'est pourquoi je propose d'explorer ici l'expérience de ces familles de deux façons, celle du récit singulier et celle des formes actuelles de son expression et de sa réception ${ }^{2}$.

\section{Une histoire familiale contemporaine}

Je vais donc commencer par évoquer l'histoire d'une famille, celle de Pauline et Stéphanie Pagès $^{3}$. Pour nombre de gens, Pauline et Stéphanie sont un cas et leur famille avec. «Etre un cas » ce n'est jamais facile car cette expression quand elle vous désigne a une fâcheuse tendance à essentialiser les choses : «être un cas » ce n'est, en effet, pas seulement se trouver dans une situation particulière, éventuellement difficile, mais c'est être quelqu'un de particulier, une personne à part. Pauline et Stéphanie ne se considèrent pas comme telles et moi non plus. Si j'ai souhaité parler ici de leur famille c'est parce qu'il est important de saisir l'expérience des individus au-delà des cas qu'ils sont censés représenter. En outre, c'est souvent la compréhension d'un parcours de vie qui est capable de donner sens à des situations ou des expériences qui au premier abord peuvent nous sembler déroutantes ou bizarres.

Stéphanie et Pauline se sont rencontrées il y a une vingtaine d'années à Nantes, l'une était étudiante en école de commerce, l'autre travaillait dans une banque. Elles se sont installées ensemble assez rapidement. A la fin de ses études, Pauline a effectué son service militaire, passage alors obligatoire pour tous les hommes de sa génération. Elles ont ensuite déménagé à Lille où Pauline avait trouvé son premier travail. Elles y sont restées trois ans et Stéphanie y a repris ses études pour préparer le concours de professeur des écoles. En 1999, elles se sont installées à Rouen où Stéphanie avait obtenu son premier poste après la réussite du concours. Pauline a saisi l'occasion pour créer sa propre société dans l'internet. C'est aussi l'année où elles se sont mariées, en deux temps et en deux lieux : d'abord à la mairie d'une ville moyenne du Sud-Ouest où réside la famille de Stéphanie, puis dans l'église d'un village normand pour répondre au vœu de la famille catholique de Pauline mais aussi pour donner un certain relief à leur mariage. Elles avaient depuis toujours le projet de fonder une famille, une famille

\footnotetext{
${ }^{1}$ En 1978, R. Green montrait que la rupture préconisée avec le parent trans était plus déstabilisante que la transition elle-même. De même, le partage des expériences cliniques entre praticiens fait que désormais aucune équipe hospitalière française n'exclut a priori les parents trans du protocole de THC.

${ }^{2}$ Pour compléter cet article, on pourra aussi regarder le court métrage de Rémy Huberdeau Transforming family sur http://vimeo.com/44615267.

${ }^{3}$ Pour préserver leur anonymat leurs noms ont été modifiés de même que tous ceux des personnes mentionnées dans le texte.
} 
nombreuse même car elles avaient dans l'idée d'avoir 5 enfants. Au seuil de la trentaine, les conditions leur semblaient réunies pour se lancer dans l'aventure. Leur projet n'a pas mis longtemps à se concrétiser car l'année suivante est né Béranger, leur premier fils. En 2002, Pauline a ouvert une filiale de sa société à Toulon car elles souhaitaient s'installer dans le Sud et près de la mer. Par les effets du rapprochement familial, Stéphanie y a obtenu un poste en 2003 alors qu'elle attendait leur deuxième enfant. Léonce est né en 2004 au moment où Pauline a fermé son entreprise pour prendre un poste de directeur administratif et financier dans une société multimédia. Elles se sont bien plu dans leur nouvelle région et ont décidé de s'y installer définitivement, matérialisant ce choix par l'achat d'une maison à La Seyne sur mer. C'est aussi à cette époque que Pauline a formulé son souhait de débuter une transition après une période de remise en question douloureuse. Les choses se sont mises en place très progressivement parce que Pauline ne voulait pas brûler les étapes de son inscription nouvelle dans la féminité, parce que Stéphanie avait aussi besoin de temps pour se positionner dans cette transformation qui bouleversait leur couple tel qu'il s'était construit jusqu'alors mais surtout parce qu'elles étaient soucieuses de la façon dont leurs enfants pouvaient appréhender la transformation de leur père. Il y a eu ainsi différentes étapes d'appropriation personnelle et familiale de la féminité de Pauline: débutée par un «travestissement clandestinisé» (localisé temporellement et spatialement), sa féminité s'est manifestée plus ouvertement et plus corporellement au fur et à mesure de la transition (orthophonie, épilation laser, hormonation, chirurgie plastique) jusqu'au moment où, comme elles le disent elles-mêmes, «on a senti que c'était prêt ». Elles ont alors décidé d'annoncer la transidentité de Pauline à la famille, aux amis et à son employeur pour qu'elle puisse enfin vivre pleinement en tant que femme. Ce «coming out » a été un moment de fragilisation pour Pauline mais aussi pour la famille toute entière. D'abord il s'est soldé par le licenciement de Pauline pour «manquements professionnels ». Côté famille et amis, les choses se sont mieux passées, il n'y a pas eu de rejet même si les réactions ont été diverses : certains ont compris et accepté « en $10 \mathrm{mn}$ » disent-elles, pour d'autres il a fallu plus de temps. Mais cette acceptation de la transidentité de Pauline a eu ses limites. Ce que Pauline et Stéphanie retiennent de ce coming out, c'est que leur couple et leur famille sont devenus, pour beaucoup de gens, suspects et illégitimes. Pauline, qui avait été toujours vu par son entourage comme un père attentionné, se sentait souvent jaugé et jugé comme si sa compétence parentale s'était brusquement évanouie. Stéphanie qui tenait à sa relation avec Pauline et n'avait jamais envisagé le divorce est apparue à certains comme une « sainte » prête à maintenir une façade conjugale (c'est-à-dire, selon eux, à renoncer à toute vie affective et sexuelle) pour préserver coûte que coûte sa famille. Pour d'autres elle était au contraire une « folle » irresponsable qui, en refusant de partir, mettait ses enfants en danger. Les enfants, quant à eux, étaient vus comme des victimes sans marge d'action, et certains loin de soutenir et d'encourager ce qu'ils mettaient en place pour s'adapter à la situation, leur disaient qu'il était regrettable qu'ils aient perdu leur père et même que son décès aurait été préférable. Les premiers psychologues consultés par Stéphanie et par les enfants n'ont pas été d'un grand secours : ils semblaient s'inscrire dans le processus de mise en cause au lieu d'être soutenants. D'autres, cependant, ont su l'être ensuite. En 2008, Pauline a entamé les démarches pour son changement d'état civil. Elle ne l'a pas obtenu car à cette époque, les tribunaux refusaient généralement le changement d'état civil aux personnes mariées (pour ne pas créer un «mariage homosexuel » de fait) et aux personnes n'ayant pas, comme Pauline, réalisé de vaginoplastie ${ }^{4}$. Elle n'a pas baissé les bras et finalement

\footnotetext{
${ }^{4}$ En France, la procédure de modification du sexe à l'état civil se fonde sur une jurisprudence établie au début des années 1990 par la cour de cassation qui impose les critères suivants pour faire droit à la demande : présenter un
} 
elle a obtenu gain de cause en 2011. Cette reconnaissance légale de l'identité féminine de Pauline a été un soulagement et une petite victoire. Il devenait possible de trouver un nouveau travail mais avant cela Pauline a souhaité reprendre ses études et elle s'est inscrite en master de marketing sous sa nouvelle identité. Parallèlement, l'idée d'agrandir la famille s'est posée mais avant d'entamer cette nouvelle étape de leur histoire, il est important de revenir sur quelques aspects de cette expérience familiale de la transition.

Des enfants et des transitions : penser et vivre leur compatibilité

Si l'expérience de Pauline et Stéphanie est singulière, elle rejoint cependant l'expérience de nombre de personnes trans qui, comme le suggère l'enquête d'A. Giami, sont majoritairement parents avant leur transition. Son enquête, réalisée en France en 2009, montre que $45 \%$ des MtF (Male to Female) et $8 \%$ des FtM (Female to Male) sont parents (Giami, 2014). Cette expérience de la parentalité est fortement liée à l'âge puisque les plus de 50 ans représentent les deux tiers des parents mais la transition apparaît aussi comme essentielle dans le devenir parent. Selon Giami, il semble que le projet parental ait été pour beaucoup un frein à la transition comme si, pour les trans aussi, il y avait là une difficile coexistence qui était négociée temporellement : d'abord les enfants puis la transition. Pour nombre d'entre eux, en effet, la transition est ou a été conçue comme potentiellement perturbante et ils ont attendu que leurs enfants soient adultes pour l'entamer au prix parfois d'une souffrance tue. D'autres cependant, comme Pauline, entament une transition avec leurs jeunes enfants mais en prenant des précautions quant à son déroulement. C'est le cas par exemple de Nathalie qui a fait ce qu'elle appelle une «transition escargot» pour que sa femme et sa fille puissent apprivoiser tranquillement sa métamorphose (Grenier, 2006). Contrairement à ce qu'on pourrait penser d'ailleurs, les enfants sont loin d'être sans ressources face à la transition de leur parent. Ils savent bien souvent lui prêter existence tout au long de la transition par le biais d'ajustements multiples. Alors que ces derniers se montrent généralement hésitants à leur imposer leur transition ou du moins réfléchissent beaucoup à la manière dont ils peuvent le faire sans les « perturber », les enfants prennent souvent les devants et provoquent les situations où leur père ou leur mère va pouvoir exprimer ce qu'il/elle souhaite être. La fille de Maryse, par exemple, a été tenue à l'écart de la transformation de son père en vertu d'un accord entre Maryse et son exfemme. Les week-ends où elle avait la garde de sa fille, Maryse avait ainsi accepté de reprendre sa présentation masculine pour ne pas la perturber. Un jour, cependant, la petite Aude a trouvé les robes de son père dissimulées dans un placard. Elle l'a alors questionné sur la propriété de ces vêtements et Maryse, ne souhaitant pas lui mentir, lui a avoué que c'était les siens. La petite fille a alors offert à son père l'opportunité de se présenter ouvertement en lui disant : «tiens, ça m'intéresserait de te voir comme ça» (Grenier, 2006, 128). Dans le même ordre d'idée, les enfants créent assez souvent, au cours de la transition de leur parent, des appellations nouvelles à son adresse afin de pouvoir associer sa position relative dans la filiation et sa nouvelle identité sexuée. La fille de Nathalie, 9 ans, a d'abord inventé «papnat » contraction de papa et Nathalie, puis «mapa» féminin de papa (Grenier, 2006, 133) pour nommer son père en transition. D'autres s'approprient le nouveau prénom pour en faire un terme d'appellation personnel comme la fille de Nadia, 9 ans, qui utilise « Nadiamounette » pour nommer son père. D'autres

syndrome de transsexualisme et avoir subi un traitement médico-chirurgical induisant des modifications corporelles irréversibles (irréversibilité associée longtemps, par les juges, à l'ablation des organes génitaux d'origine). 
encore, comme la fille adolescence d'Adrien Blanchet, contextualise les appellations : « elle m'appelle toujours maman mais devant des inconnus elle dit Adrien ». Ces ajustements nous montrent qu'un statut parental ne renvoie pas uniformément à une inscription sexuée particulière, mais qu'il dépend tout autant de l'histoire familiale et des relations de parenté qui $\mathrm{y}$ ont pris forme. Dans ces familles, comme dans les autres d'ailleurs, être père ou mère est le résultat d'une histoire qui réfère aux dimensions tout à la fois corporelle, affective et sociale des relations interindividuelles. On peut être ainsi femme et père ou homme et mère sans qu'il y ait là de discordance identitaire mais simplement les effets d'un parcours de vie.

\section{Une histoire familiale (suite)}

Après le changement d'état civil, le souhait d'agrandir la famille est réapparu. Pauline et Stéphanie l'avaient toujours envisagé et d'ailleurs quand Pauline avait commencé l'hormonation, elles avaient pensé faire une autoconservation de sperme dans cet objectif. Elles avaient aussi pensé à l'adoption mais leur situation ne semblait pas leur donner les meilleurs chances d'obtenir l'agrément nécessaire: Pauline était alors sans emploi et sa transition était en cours. Même si désormais la situation était différente, elles pensaient n'avoir pas plus de chances de l'obtenir au vu des réactions ordinaires à leur famille. Elles ont donc choisi de faire cet enfant de la même façon que les deux premiers. Cela supposait que Pauline arrête les hormones pour que la spermatogénèse se rétablisse mais cet arrêt n'était pas sans les questionner : Stéphanie avait peur que Pauline se sente mal et elles craignaient aussi que la situation ne leur échappe car les endocrinologues consultés n'avaient aucune expérience en la matière. Elles se sont demandé si le jeu en valait la chandelle. Finalement elles ont décidé d'essayer et il leur a fallu presque un an pour qu'une grossesse se profile. Malheureusement au bout de trois mois, l'échographie a révélé un fœus sans vie que Stéphanie a quand même dû porter encore 5 semaines avant qu'une fausse couche ne se déclare. Cette période où elle savait son enfant mort en elle a été très éprouvante surtout que parallèlement leur entourage ne comprenait pas du tout leur projet. On leur disait qu'elles étaient complètement inconscientes de l'avenir difficile qu'aurait nécessairement cet enfant, qu'elles avaient déjà bien assez de soucis avec les deux aînés qui ne manqueraient d'ailleurs pas d'être choqués par cette naissance. D'autres aussi découvraient, à la faveur de cette grossesse, que la sexualité du couple, qu'ils avaient pensée inexistante, était loin de l'être et derrière leur «comment c'est possible? ? pointait un certain effarement. Malgré cet accueil négatif de leur projet, elles n'ont pas hésité à faire un nouvel essai et finalement Stéphanie a été de nouveau enceinte. Leur petite fille, Marie Lou est née en mars 2014 mais elle n'a légalement qu'un seul parent, Stéphanie, car l'officier d'état civil a refusé de prendre en considération l'acte de reconnaissance anticipée de Pauline. Pour lui, la présomption de paternité ne s'applique pas puisqu'il s'agit d'un mariage entre deux femmes. Pour que le lien de filiation entre Pauline et Marie-Lou soit établi il faudrait que Pauline adopte l'enfant de sa femme comme peuvent désormais le faire les lesbiennes mariées. Mais cette solution heurte Stéphanie et Pauline car cela instaure une différence entre leurs enfants qui ont pourtant été tous engendrés de la même façon. Une nouvelle bataille judiciaire s'est donc engagée pour la reconnaissance du lien de filiation entre Pauline et Marie-Lou.

«L’engendrement trans » : une réalité matière à controverse 
Pendant longtemps il a été impossible pour les personnes trans d'envisager avoir des enfants après la transition du fait des conséquences induites par les opérations génitales réalisées. Désormais les techniques de PMA permettent de rendre possible cette parentalité post-transition que ce soit via des Inséminations avec donneur ou encore via l'autopréservation des gamètes avant THC. Au plan international, la santé reproductive des personnes trans et notamment la préservation de leur fertilité est d'ailleurs prise en considération depuis une décennie comme le suggèrent les Standards de soins de la WPATH ${ }^{5}$ qui encouragent l'information des patients et la mise en œuvre de la conservation des gamètes. Mais ces projets parentaux post-transition ne rencontrent pas un égal accueil de la part des équipes médicales que ce soient celles qui sont spécialisées dans la prise en charge des TIG ou celles qui se consacrent à la médecine reproductive. Pour un certain nombre de médecins, la question de l'engendrement des personnes trans, semble poser problème. En France, l'accueil des hommes trans dans les $\operatorname{CECOS}^{6}$ a entraîné une réflexion éthique notable depuis deux décennies, de même que les demandes d'autopréservation de sperme des femmes trans suscitent actuellement la controverse.

Contrairement à ce que l'on pourrait croire, l'accueil de personnes trans dans les CECOS pour une IAD n'est pas récent bien qu'il émerge récemment hors de la stricte sphère des équipes médicales concernées. En l'absence d'enquête précise, il est difficile d'avoir une idée du nombre de demandes d'aide à la procréation émanant de personnes trans en France. Les données dont nous disposons, sont celles qui proviennent de l'équipe de l'Hôpital Cochin à Paris qui a publié récemment ses résultats ${ }^{7}$. Cette équipe a reçu 86 demandes $^{8}$ émanant de couples formés d'un homme trans et d'une femme cisgenre ${ }^{9}$. Selon P. Jouannet (2014) la première demande a été faite en 1987 et a été traitée comme celle des autres couples reçus habituellement; elle a permis la naissance de deux filles, aujourd'hui âgées d'une vingtaine d'années. À partir du milieu des années 1990, les demandes se sont multipliées et l'équipe s'est posé la question de la prise en charge de ces couples, ce qui a donné lieu à une réflexion au sein de l'Espace éthique de l'Assistance publique-Hôpitaux de Paris (AP-HP). De manière générale, les réticences qui ont été à l'origine de cette réflexion médico-éthique réfèraient, d'une part, à la conception pathologique classique des personnes trans et, d'autre part, à la mise en doute de leur capacité à s'inscrire de manière adéquate dans la filiation et la parentalité. Elles renvoyaient ainsi à des interrogations sur la capacité paternelle de ces hommes (seront-ils des pères ? seront-t-ils de bons pères ?) et sur le développement psycho-affectif et l'inscription sexuée de leurs enfants (seront-ils «atteints » eux aussi de TIG ? seront-ils des garçons et des filles normaux ?). À l'issue de ce processus, un protocole spécifique «expérimental » a été mis en place pour l'accueil de ces couples comprenant une consultation supplémentaire avec un psychiatre ayant l'expérience du TIG et un suivi clinique des enfants tous les deux ans devant permettre à moyen ou long terme de dresser un bilan raisonné.

Pour répondre au cadre légal définit par la loi de bioéthique de $1994^{10}$, le CECOS de Cochin n'a pris (et ne prend) en considération que les demandes des couples où le changement

\footnotetext{
${ }^{5}$ World professional association for transgender health qui publie des standards de soins internationaux depuis la fin des années 1970 dans lesquels est inclu, depuis la $6^{\text {ème }}$ version de 2001, un chapitre sur la santé reproductive des personnes trans.

${ }^{6}$ Centre d'étude et de conservation des œufs et du sperme humains chargé de la gestion des dons de gamètes.

${ }^{7}$ En l'absence d'autres travaux, nous nous centrerons sur les publications de cette équipe.

${ }^{8}$ Sur ces 86 demandes, 20 n'ont pas été confirmées et 5 ont été refusées. Parmi les 57 couples qui ont débuté une Insémination avec donneur (IAD), 32 ont eu au moins un enfant et 46 enfants étaient nés en 2012 (Jouannet, 2014).

${ }^{9}$ Formé à partir du préfixe cis- (antonyme de trans-) qui sert à désigner ce qui est en deçà ou dans la limite de, le terme cisgenre est utilisé de plus en plus souvent pour qualifier les personnes ou les expériences «non-trans ».

${ }^{10} \mathrm{Ne}$ peuvent être acceptés que les couples hétérosexuels mariés ou pouvant justifier d'au moins deux ans de vie commune, en âge de procréer et dont l'infertilité a été médicalement diagnostiquée
} 
d'état civil de l'homme a été prononcé. Cette décision qui n'est que le corolaire d'une disposition légale renvoie cependant à des implicites qu'il est important d'explorer. Les exigences des juridictions pour prononcer un changement d'état civil ont eu un effet notable : la majorité des hommes trans légalement reconnus de sexe masculin ont subi, de façon volontaire ou contrainte, une hystérectomie et une ovariectomie. Ces dernières années cependant, l'exigence de stérilisation pour l'obtention du changement d'état civil n'est plus aussi systématique (pour diverses raisons qu'il serait trop long de développer ici), si bien que certains hommes n'ont pas subi les opérations citées. Cela a d'ailleurs été le cas de deux hommes ayant fait une demande d'IAD avec leur compagne. Contrairement aux autres, ces hommes n'était donc pas «irréversiblement»stériles mais la testostérone qu'ils prenaient régulièrement les rendait bien tels et, en outre, ils ne pouvaient de quelque manière que ce soit faire un enfant avec leur partenaire. En ce sens, ils répondaient bien à l'exigence légale d'une infertilité médicalement diagnostiquée ce que d'ailleurs l'équipe médicale ne semble pas avoir remis en question. Pourtant, elle a été semble-t-il perturbée par leur situation au point de leur demander de réaliser une hystérectomie-ovariectomie (ce qu'ils ont fait apparement sans problème) avant d'entamer l'IAD dont ils étaient demandeurs. On voit donc l'hystérectomieovariectomie devenir ici, très paradoxalement, un critère d'inclusion dans un protocole de PMA.

Les résultats du suivi des couples et des enfants qui ont été publiés en 2013 semblent « rassurants ». Ils montrent que les hommes trans s'inscrivent dans la paternité de façon exclusive et qu'il n'y a, pour eux et pour leurs enfants, aucune ambiguïté quant à leur rôle parental. Les enfants sont dits normaux et cette normalité est soigneusement détaillée et réitérée notamment pour ce qui concerne la sexuation : «Pour l'équipe psychologique qui les a vus, ce fut un plaisir de recevoir des enfants « normaux », tels qu'on souhaite en avoir, sans retard de développement, sans symptômes inquiétants, qui sont vraiment des garçons ou des filles sans (...) dysphorie de genre. (Chiland et al., 2013 : 114). Pourtant, contrairement à ce qu'on aurait pu attendre au vu de ces résultats, ce n'est pas l'arrêt de l'expérimentation qui est envisagé mais plutôt sa pérennisation. Autrement dit, cette expérience n'aboutit pas à proposer un traitement non spécifique des personnes trans ni même à l'arrêt du suivi des enfants (dont les effets sur ces derniers sont peu discutés) mais bien au contraire à la validation d'une modalité de traitement spécifique des personnes trans en PMA. En une décennie, l'objectif initial semble ainsi s'être transformé : il ne s'agit plus tant de lever des réticences initiales multiples et de favoriser l'ouverte de la PMA aux personnes trans mais plutôt de pérenniser, voire de généraliser, une prise en charge discriminante de personnes dont on dit pourtant qu'elles font famille normalement.

On se rappelle que Pauline et Stéphanie avaient envisagé une conservation de sperme au moment de leur nouveau projet d'enfant. Elles n'ont pas choisi de poursuivre dans cette voie mais d'autres femmes trans l'ont fait récemment ${ }^{11}$. Les CECOS sollicités ont refusé toutes les demandes si bien que les intéressées, soutenues par des associations, ont mobilisé le Défenseur des droits, envisageant la réponse des CECOS en termes de discrimination au vu du Code de la Santé publique (art L2141-11) qui précise que « toute personne dont la prise en charge médicale est susceptible d'altérer la fertilité (...) peut bénéficier du recueil et de la conservation de ses gamètes ». Avant de donner un avis, le Défenseur des droits a proposé une saisine de différentes instances médicales notamment l'Académie nationale de médecine et l'Agence de biomédecine qui ont rendu leur avis courant 2014. Les deux rapports vont dans le même sens, statuant qu'il n'y a pas lieu de faire droit à ces demandes. L'Académie de médecine notamment considère

\footnotetext{
${ }^{11}$ En 2014, l'Académie de médecine mentionnait une quinzaine de demandes d'autoconservation de sperme des
} femmes trans faites auprès des CECOS au cours des dernières années (Académie nationale de médecine, 2014). 
qu'il n'y a pas d'indication médicale à cette conservation et que l'usage ultérieur des gamètes, s'il est techniquement possible, n'est ni actuellement légal ni surtout souhaitable : les filiations qui seraient ainsi établies seraient socialement bouleversantes et psychiquement délétères (notamment pour les enfants). Les craintes évoquées ici relèvent de ce qu'on peut appeler « l'effet Thomas Beatie » du nom de cet américain qui a porté et mis au monde ses trois enfants après sa transition et son changement d'état civil ${ }^{12}$. L'expérience de cet homme a été assez généralement comprise comme déstabilisant les fondements de la parenté et le rapport de l'Académie de médecine essaie de montrer qu'il en est de même pour ce qui concerne l'usage du sperme autoconservé des femmes trans. Pourtant, les expériences d'engendrement de Pauline, de T. Beatie et de bien d'autres encore ne contreviennent pas fondamentalement aux attentes communes. Elles sont certes inédites mais pas renversantes. Et pour saisir cela il faut simplement comprendre que l'identité des personnes n'est pas seulement une question de propriétés mais tout autant une question d'histoire et de relations. Définir/décrire une personne ce n'est pas simplement en effet faire l'inventaire de ses caractéristiques mais c'est être capable de relater une histoire, la sienne (qui la définit effectivement dans sa singularité), et de rendre compte des liens multiples et variés qui l'attachent aux autres et qui la font exister dans un univers social particulier. L'histoire corporelle des femmes trans n'est ainsi en rien discordante avec leur inscription sexuée et tout un chacun est capable de le saisir sans changer pour ce faire de biologie: leur prostate et leurs spermatozoïdes ne sont pas des traits « discordants » mais simplement des indices de la façon dont elles se sont inscrites dans la féminité (c'est-à-dire via une transition). De même «l'engendrement trans » n'est guère bouleversant si l'on veut bien le décrire dans le langage des actions et des relations (c'est-à-dire en termes d'histoire et de manière d'agir) plutôt qu'en termes de propriétés. Une femme trans qui utilise son sperme pour avoir un enfant n'est pas ce père-mère si souvent craint parce qu'il amalgamerait les statuts parentaux, simplement elle engendre à la manière des hommes en tant que femme, ce qui fait d'elle une mère dans notre système de parenté ${ }^{13}$. Elle agit à la manière des hommes non pas seulement parce qu'elle utilise son sperme (un matériau «biologique ») mais parce qu'elle se positionne dans cet acte d'engendrement dans un rôle habituellement tenu par les hommes : celui de donner à d'autres, dans un cadre relationnel socialement signifiant, une substance permettant de mettre au monde un être humain. Cet «agir à la manière de » ne fait pas d'elle un père. Comme tout un chacun, ce qui fait d'elle un parent, c'est son engagement « en tant que ", engagement qui est corrélatif de ses autres statuts dans l'ordre de l'affinité et de la consanguinité (épouse, sœur, fille, etc.). Il n'y a pas ici d'amalgame identitaire (le fameux « père-mère ») mais seulement des registres d'action différents : agir à la manière de et agir en tant que étant des étiquettes qui annoncent le sens de nos actions et les rendent intelligibles. Autrement dit, l'engendrement trans qui effraie souvent à tort ne met à mal ni notre biologie, ni notre système de filiation et pour le comprendre il faut cesser de penser le genre comme une simple propriété des personnes.

A l'issue de cette approche des transparentalités contemporaines, on peut dire que l'avenir des familles trans reste difficile à cerner : à la fois plein de promesses d'existence et plein d'obstacles sociaux et de veto institutionnels. Mais une chose semble sûre, les personnes trans ne sont plus prêtes à renoncer à leurs projets parentaux et c'est sans doute pourquoi il est important de se rappeler que ceux-ci ne renversent pas notre monde comme nous sommes souvent tentés de le croire. Simplement ils accroissent sa diversité et ils nous invitent à réfléchir

\footnotetext{
12 Pour une analyse de son expérience et de sa réception, cf. l'ouvrage collectif intitulé la parenté transgenre (Hérault, 2014a).

${ }^{13}$ Pour une présentation plus développée de cette description cf. (Hérault, 2014b).
} 
aux possibilités d'existence que nos sociétés se montrent capables d'offrir à ces personnes et à leurs familles.

\section{Bibliographie}

Académie nationale de médecine. 2014. Autoconservation des gamètes des personnes transsexuelles et projet parental éventuel. Paris, Académie nationale de médecine.

Chiland, C., Clouet, A.-M., Guinot, M., Golse, B., Jouannet, P., \& Revidi, P. 2013. « Pères d'un nouveau genre et leurs enfants ». Psychiatrie de l'enfant, Vol. 56(1), p. 97-125.

Cordier B., Chiland C., Gallarda T., 2001 Le transsexualisme, proposition d'un protocole malgré quelques divergences, Annales Médico-Psychologiques, 159 : 190-195.

Giami, A. 2014. «Procréation et parentalité dans la population trans. Genre, parcours biographique, parcours de transition ». in L. Hérault (dir), La parenté transgenre. Aix-en-Provence, Presses Universitaires de Provence p. 93-105.

Green, R., Money, J., 1969 Transsexualism and Sex Reassignment. Baltimore, John Hopkins Press

Green, R., 1978 Sexual identity of 37 children raised by homosexual or transsexual parents. American Journal of Psychiatry, 135, 692-697.

Grenier M. 2006, «Papa t'es belle ». Approche anthropologique des paternités transsexuelles. Mémoire de Master 2 d'anthropologie, Aix-en-Provence, Université de Provence.

Hérault, L. (dir.) 2014a. La parenté transgenre. Aix-en-Provence, Presses Universitaires de Provence.

Hérault, L. 2014b. «Procréer à la manière des femmes, engendrer en tant qu'homme ». in L. Hérault (dir), La parenté transgenre. Aix-en-Provence, Presses Universitaires de Provence, p. 79-90.

Jouannet, P. 2014. «Quand les hommes transsexuels souhaitent devenir pères par don de sperme ». in L. Hérault (dir), La parenté transgenre. Aix-en-Provence, Presses Universitaires de Provence p. 117-125.

World Professional Association for Transgender Health. 2007. Standards of care for the health of transsexual, transgender and gender non conforming people. 7th version. WPATH. 